\title{
In-situ Icing and water condensation observation on different topographical
} surfaces

Halar Memon ${ }^{a}$, Junpeng Liu ${ }^{a}$, Nicola Weston ${ }^{b}$, Jie Wang ${ }^{a}$, Davide De Focatiis ${ }^{a}$, Kwing-so Choi ${ }^{\mathrm{a}}$ and Xianghui Hou ${ }^{\mathrm{a},{ }^{*}}$

aFaculty of Engineering, University of Nottingham, University Park, Nottingham NG7 2RD, UK;

${ }^{b}$ Nano and Micro Research Centre (nmRC), University of Nottingham, University Park, Nottingham NG7 2RD, UK;

*Correspondence: xianghui.hou@nottingham.ac.uk;

\section{Abstract}

Icephobicity is intrinsically affected by rough asperities and the surface voids provide anchoring points for the ice. The anchor of ice is likely to form on the surface under high humidity conditions. In-situ water condensation and icing observation were conducted to understand water condensation and ice retracting patterns in controlled humidity, pressure and temperature conditions. It was observed that water microcondensation and icing occurred on rougher surfaces and the water droplets condensed along the surface cracks of the superhydrophobic polydimethylsiloxane (PDMS) based nanocomposite coatings. Further analysis revealed that ice anchoring was present on both aluminum and superhydrophobic coating surface, but it was more severe and intensified on the as-received aluminum substrates. No water condensation or subsequent icing was found on smooth PDMS hydrophobic surfaces due to the incapacity of the smooth surfaces to anchor water drops. It is the first time to validate ice anchoring over retracting ice on different wettability surfaces from insitu icing observation. Ice adhesion strengths were also measured on the studied 
surfaces and the results indicated a strong linkage between centrifugal shearing of ice and anchoring mechanism due to surface rough voids, and there was no clear relevancy between ice adhesion strength and the surface wettability or hydrophobicity.

Keywords: icephobicity, superhydrophobicity, ice anchoring, in-situ icing

\section{Introduction}

For decades, the idea of deploying superhydrophobic surfaces for icephobic performance was studied and widely experimented [1-4]. Superhydrophobic surfaces practically suspend the water droplets in Cassie-Baxter wettability status which minimizes the surface contact by suspending the water droplets on the air pockets or void valleys of the surface [5] and reduces the possibility of anchoring of water on the surface asperities [6]. The principle behind the use of superhydrophobic surfaces for icephobic applications is to freeze the water droplets in the Cassie-Baxter stage, sometimes also referred as 'Cassie ice', and form the weak bonding of ice on the surfaces [7-9]. Intrinsically, the surface can be functionalized into hydrophobic by chemical modifications with low surface energies and it was reported that receding water contact angle of $\sim 120^{\circ}$ can be achieved via chemical modifications made by Carbon fluoro and/or silane-based chemicals $[10,11]$. Superhydrophobic surface is mainly achieved by the combined effects of low surface energy modification and rough asperities [12-15]. However, in some occasions, the introduction of rougher asperities on the surface (which renders the surface superhydrophobicity) $[16,17]$ leads to higher ice adhesion strength and require higher stress to break the ice on the surface with complex topographical features $[4,18]$. Zou et al [19] reported that water contact angle (WCA) changed from $83^{\circ}$ on aluminum surface to $37^{\circ}$ after sandblasting. However, a further modification of these aluminum surfaces with fluorinated-carbon molecules 
resulted in water contact angles of $117^{\circ}$ and $145^{\circ}$ for the untreated $\mathrm{Al}$ and sandblasted

Al samples respectively. Ultra smooth surfaces $(<10 \mathrm{~nm})$ have also attracted some attention in the anti-icing study. Jung et al [1] reported 150-times freezing delays on surface having nano-scale roughness. It is suggested [20] that roughness near to ice nuclei scale is particularity favorable for the anti-icing performance. Mishchenko et al used highly ordered nano-sized surfaces $(\mathrm{Ra} \approx 0.17 \mathrm{~nm})$ and demonstrated the delay of ice formation for remarkable 25 hours [21].

Liu et al [22] used fluoroalkyl silane lubricated nano silicon oxide deposited surfaces and demonstrated water contact angles of $163^{\circ}$. In terms of icephobic performance, they reported water droplet icing delay (under static conditions) of 289 seconds in comparison to the reference substrate which formed ice in just 29 seconds. They further claimed a twofold decrease in ice adhesion strength as a comparison to pristine substrates. Hancer et al [23] combined polysilicon (silsesquioxane) matrix with $12 \mathrm{~nm}$ $\mathrm{SiO}_{2}$ nanoparticles and the nanoparticles were rendered hydrophobic by chemical modification using a self-assembled monolayer of perfluorodecyltrichlorosilane. Near theoretical superhydrophobicity of $178^{\circ}$ was reported at $3 \mathrm{wt} \%$ of nanoparticles to polymer matrix and droplet bouncing and sliding behavior at $-20{ }^{\circ} \mathrm{C}$ ambient temperatures was demonstrated. Cao et al [24] synthesized superhydrophobic polymer nanocomposite using acrylic polymer by free radical polymerization and reported no indication of ice accretion on superhydrophobic surfaces was observed at sub-zero temperatures.

However, there are certain limitations in use of superhydrophobic surfaces for icephobic performance and this hypothesis is valid until micro frost formation occurs, for example, high-speed impingement of water droplets would wet the rough asperities of surface and form micro condensation of water [6]. Under sub-zero temperature, the 
micro condensation forms a thin layer of ice which effectively nullifies the

superhydrophobicity of surfaces [25, 26]. Murphy et al. [27] studied dynamic defrosting on superhydrophobic surface and found that frost did form over both hydrophobic and superhydrophobic surfaces. However during thermal de-icing, the melted water droplets over the superhydrophobic surface was more mobile as compared to those on the hydrophobic smooth polymers, as the superhydrophobicity of the surface was restored after the removal of frost. It is reported that superhydrophobic surfaces have promising icephobic performance down to $-20^{\circ} \mathrm{C}$ to $-30^{\circ} \mathrm{C}[21,24,28]$. But under high humidity conditions, the icephobicity of superhydrophobic surfaces is deteriorated due to capillary action of surface asperities and micro condensation which leads to ice build-up and/or the changes of the wetting model to Wenzel configuration from CassieBaxter configuration [29, 30].

In the present work, in light of these experimental results and assumptions, in-situ icing observations will be applied to acquire direct evidence of ice anchoring over rough asperities of superhydrophobic/ aluminum under high humidity conditions. The assumptions of a water anchoring mechanism over rougher surfaces will be validated regardless of the surface wetting conditions via in-situ water condensation and icing observations. A combination of a high humidity, sub-zero temperature, and low pressure environment are ideal conditions for icephobicity tests in which the extreme environmental conditions would be simulated for the development of passive ice protection system for aviation applications.

\section{Experimental}

Five different material/coating types were used in this study: Pristine as received aluminum substrates (AR-Al), smoothened aluminum substrates (S-Al), sandblasted 
aluminum substrates (SB-Al), polydimethylsiloxane (PDMS) coating on aluminum substrates and PDMS silicon oxide nanocomposite coatings on aluminum substrates (Nano-SiO $/$ PDMS).

\subsection{Substrates and raw materials}

Two part PDMS polymer R-2180 was procured from NuSil technology LLC and hydrophobic functionalized silicon oxide nanoparticles were purchased from Evonik AEROSIL. Aluminum 2024 (Al2024-T4) plates of size $50 \mathrm{~mm} \mathrm{X} 20 \mathrm{~mm} \mathrm{X} 3 \mathrm{~mm}$, were used as observation surfaces and coating substrates.

The AR-Al samples were washed thrice with ethanol and deionized water and dried using compressed air. The S-Al samples were smoothened using grinding and polishing with a series of steps employing sandpapers having grits sizes of 220, 320, 400 and $600,1 \mu \mathrm{m}$ polishing cloths, and $0.25 \mu \mathrm{m}$ (chemically induced) polishing cloths using Metprep colloidal silica suspension particles, respectively. The SB-Al samples were roughened using Guyson F1200 sandblaster system using Guyson 180-220 $\mu \mathrm{m}$ alumina particles. All of the samples were washed and dried before use.

\subsection{Preparation of coatings}

For PDMS coatings, 1 gram of PDMS Part A and Part B (1:1) were mixed in $3 \mathrm{ml}$ xylene using magnetic stirring for 3 hours. For $\mathrm{Nano}_{-} \mathrm{SiO}_{2} / \mathrm{PDMS}$ coatings, 1 gram of PDMS Part A and Part B were mixed (80\% wt) in $1 \mathrm{ml}$ xylene using magnetic stirring for 3 hours and 0.5 gram nano- $\mathrm{SiO}_{2}$ were dispersed (20\% wt) in $2.5 \mathrm{ml}$ xylene using ultrasonic mixing for one hour. Both the solutions were then mixed using magnetic stirring for one hour. 
120 The coatings were applied on SB-Al samples using Chemat Technology spin-coater $\mathrm{kW}-4 \mathrm{~A}$ at a rotation speed 1500 RPM for one minute and were dried and cured at 60 ${ }^{\circ} \mathrm{C}$ for 60 minutes, and then $150{ }^{\circ} \mathrm{C}$ for 150 minutes.

\subsection{Hydrophobicity and icephobicity tests}

Water contact angles (WCAs) were measured using the sessile drop technique on FTÅ200 goniometer and the volume of one water drop was kept constant at $5 \mu$. Dynamic WCAs (advancing and receding angles) were measured using dispense dip method. $5 \mu \mathrm{l}$ drop was suspended over the surface and the dispense dip was immersed in the droplet. Advancing and receding WCAs were measured when the base length of the droplets were increasing or decreasing constantly. Flow rate of the water was kept constant at $1 \mu \mathrm{l} / \mathrm{s}$ and dynamic WCAs values were measured as the average of 5 consecutive values. Contact angles hysteresis $(\mathrm{CAH})$ was calculated from the difference of advancing WCAs to receding WCAs. The tests were conducted at room temperature and humidity conditions.

Ice adhesion strength tests were conducted using the centrifugal method in a $500 \mathrm{~mm}$ diameter drum via MOOG G403-2053A servo motor and the equipment was kept in a

Ice adhesion strength of ice can be calculated by,

Design Environmental ALPHA 1550-40H (environmental chamber) to mimic the icing conditions. A controlled volume of de-ionized water was filled in silicone molds, the molds were then flipped upside down and kept against gravity for overnight freezing at $-10^{\circ} \mathrm{C}$. The samples were then mounted on a carbon fiber reinforced arm via screws and spun at a rotation speed up to $4500 \mathrm{rad} / \mathrm{min}$ at $30 \mathrm{rev} / \mathrm{min} / \mathrm{sec}$ acceleration $(3.14$ radian/second ${ }^{2}$ ). The ice adhesion test was conducted at a temperature of $-10{ }^{\circ} \mathrm{C}$.

$$
F=m r \omega^{2}
$$


144 Where $\omega$ is the rotational speed $(\mathrm{rad} / \mathrm{s})$ at removal, $r$ is the rotor length and $\mathrm{m}$ is the mass of ice. Shear removal stress can be calculated by,

$$
\tau_{\text {ice }}=F / A
$$

147 Where $A$ is the substrate/ice contact area and $F$ is the centrifugal shearing force.

\subsection{In-situ observation and surface characterization}

The microstructural analysis and In-situ icing and condensation observations were

150 carried out using a FEI Quanta650 eSEM system. The system is capable of generating micro-level HD surface images, under controlled humidity and temperature by a Peltier cooling stage. The chamber humidity (above $90 \% \mathrm{RH}$ ) was raised to wet the coating surface and in-situ water condensation was studied. Secondly, the condensed water on top of the coating surface was frozen at a temperature of $-5^{\circ} \mathrm{C}$ and high humidity $(85-95 \% \mathrm{RH})$ conditions. The retracting pattern of the formed grown ice was analyzed.

The surface roughness was evaluated out using a Zeta-20 non-contact optical profiler. 100x magnification was used for surface roughness measurements and $5 x$ magnification was used for 3D surface profiling images. Surface roughness values in this study were based on the average of several measurements.

3. Results and discussion 


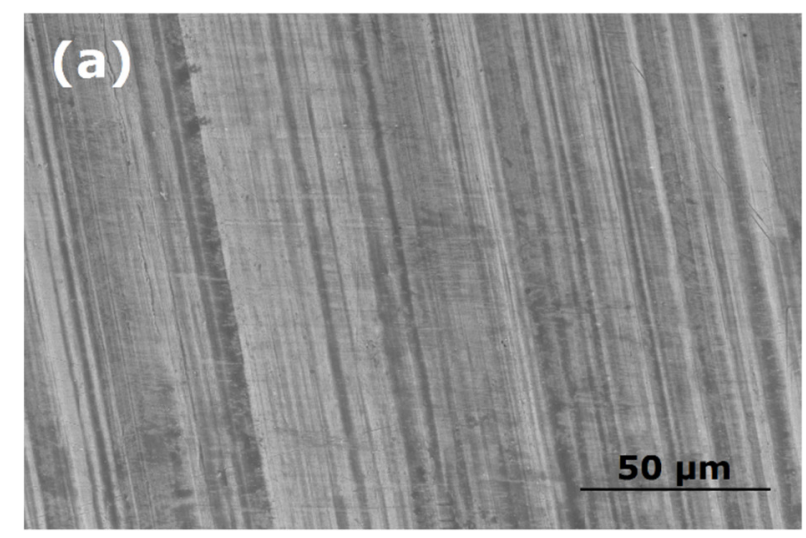

(c)

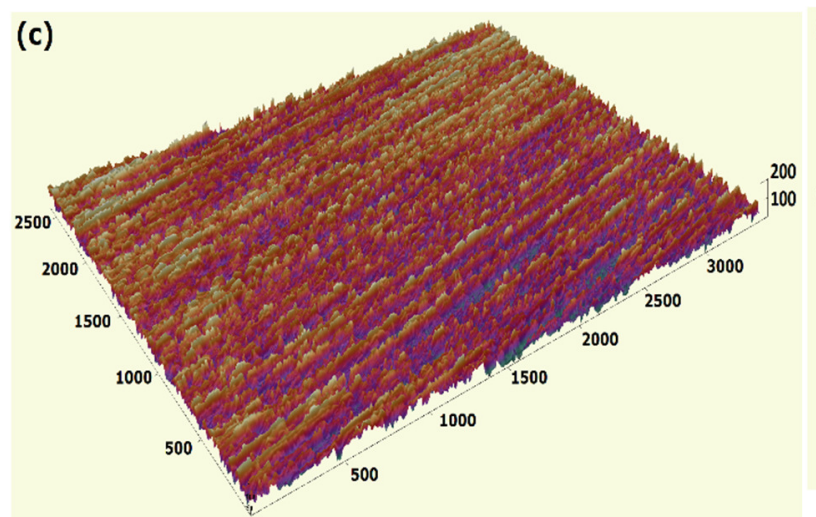

AR-Al substrates have relatively high elastic modulus (72.4 GPa [31]) as compared with PDMS (2.4 MPa [32]), and have surface topographic pattern ( $\left.R_{a} 0.9 \mu \mathrm{m}\right)$ as shown in Figure 1a and 1c. AR-Al substrates surface has a considerable heterogeneous solid surface (rougher asperities) [33, 34], which may acts as icing seeds by reducing activation energy for ice nucleation [35].

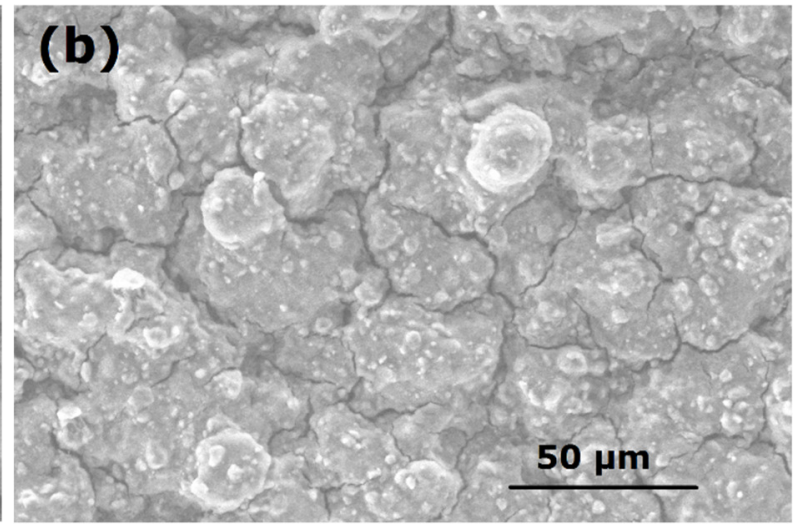

(d)

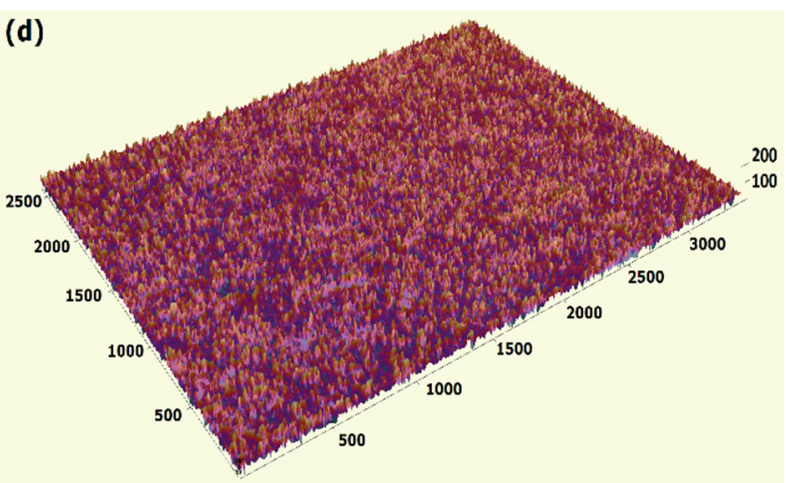

Figure 1 (a) SEM image of AR substrates, (b) SEM image PDMS-Nano $\mathrm{SiO}_{2}$ coatings, and (c) 3D surface profile of AR substrates, (d) 3D surface profile of PDMS-Nano $\mathrm{SiO}_{2}$ coatings

The Nano-SiO $/$ PDMS coatings on aluminum substrates behaved in a superhydrophobic manner with a rough surface, and had a medium elastic modules of approximately 9.4 MPa [36]. These coatings were exceptionally rough surfaces $\left(\sim R_{a}\right.$ $1.9 \mu \mathrm{m})$ as shown in Figure $1 \mathrm{~b}$ and $1 \mathrm{~d}$ and rough voids present on the coating surface were favorable to the superhydrophobic performance [22] but the cracks were prominent and the cracks might be induced because of incorporation of silicon oxide 
nanoparticles. It is hypothesized that reduction in ice adhesion strength is possible with high levels of surface roughness as it increases the number of air pockets presented between the inter-facial ice-substrate contacts, thus reducing the contact area of ice/surface interface $[5,7,37]$. However, surface roughness also increases the number of possible anchoring sites, which may lead to higher adhesion strengths in some instance [33] or increasing the amount of energy required to break the adhesion among the highly unordered rough voids $[4,25]$.
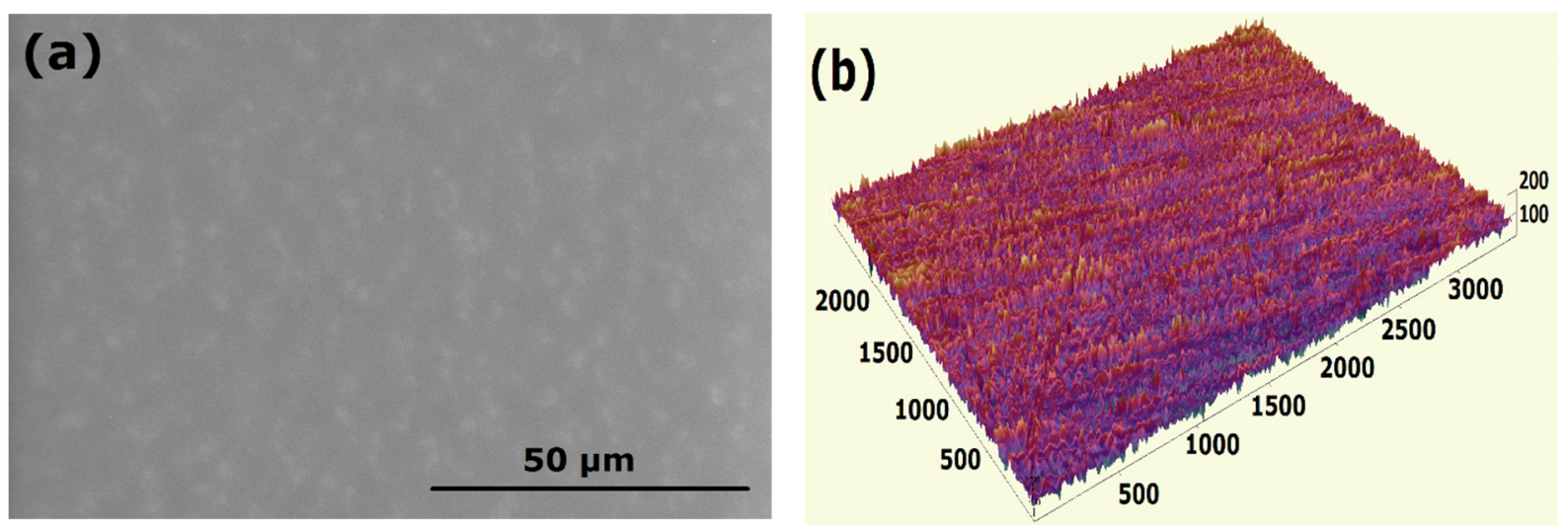

Figure 2: (a) microstructural image and (b) 3D surface profile of PDMS coatings The selection of sample surfaces was entirely made to have in-situ icing and observations on surfaces having different wettability and surface texturing. Static and dynamic water contact angles and ice adhesion strength measured on the examined surfaces are summarized in Table 1. AR substrates behaved in a hydrophilic manner and demonstrated high $\mathrm{CAH}$ and ice adhesion strength. Smoother PDMS coatings $\left(\sim R_{a} 0.12 \mu \mathrm{m}\right)$ is indicated in Figure $2 \mathrm{a}$ and $2 \mathrm{~b}$ and low $\mathrm{CAH}$ and ice adhesion strength were measured. Obtained results of $\mathrm{CAH}$ and ice adhesion strength are in good agreement with the observation of Zaid et al [38] that low ice adhesion strength can be achieved when the $\mathrm{CAH}$ value is around $25^{\circ}$ but contradictory to several studies [ 8 , 39-42], which links low CAH to lower ice adhesion strength. The present results indicated that the lowest ice adhesion strength on PDMS coatings, whereas the lowest 
$191 \mathrm{CAH}$, was found on $\mathrm{Nano}-\mathrm{SiO}_{2} / \mathrm{PDMS}$ coatings. Nano-SiO$/ 2 / \mathrm{PDMS}$ coatings behaved in a superhydrophobic manner and this could be primarily attributed to the rough morphology and low surface energy. It is widely accepted that superhydrophobicity could only be realized by inducing certain surface roughness either by nanoparticles $[23,43]$ or controlled surface roughness [21]. For Nano-SiO$/ 2 / \mathrm{PDMS}$ coatings, as the chemical composition of PDMS and the nanoparticles used are hydrophobic and the combined effect renders the surface superhydrophobic [22].

Ice adhesion strength results on AR substrates and $\mathrm{Nano}-\mathrm{SiO}_{2} / \mathrm{PDMS}$ coatings gives us the idea that the surface energies and elastic modulus play a prominent role on icephobic performance as both were not smooth samples but varied in surface energies and elastic modulus. It is suggested that low surface energy had contributed to low surface wettability in varies studies [44-46]. It can also be assumed that PDMS based coatings were low modulus elastic in nature and this elasticity could have induced interfacial cavitation mechanism. Thus, the smoother topography of PDMS based coating could have played a deciding factor in icephobicity $[18,47]$. It is believed that an ultra-smooth surface with a layer of low surface energy liquid at the interface would nullify the effect of surface asperities and impart icephobicity [47]. Stamatopoulos et al. [48] demonstrated that a self-impregnating slippery surface is able to delay the ice formation by $2-3$ folds and reported reduction in ice coverage by 10-15 times as compared to superhydrophobic and smooth surfaces. However, the durability and liquid retention are the major concerns in the liquid containing slippery coatings [49]. 
214 Table 1: Wettability, icephobicity, and surface roughness results of experimented 215 materials

\begin{tabular}{|l|l|l|l|l|l|l|}
\hline $\begin{array}{l}\text { Coating } \\
\text { Types }\end{array}$ & $\begin{array}{l}\text { Static } \\
\text { WCAs } \\
\left(^{\circ}\right)\end{array}$ & $\begin{array}{l}\text { Advancing } \\
\text { WCAs }\left(^{\circ}\right)\end{array}$ & $\begin{array}{l}\text { Receding } \\
\text { WCAs }\left(^{\circ}\right)\end{array}$ & $\begin{array}{l}\text { CAH } \\
\left(^{\circ}\right)\end{array}$ & $\begin{array}{l}\text { Ice } \\
\text { adhesion } \\
\text { strength } \\
(\mathrm{KPa})\end{array}$ & $\begin{array}{l}\text { Roughness, } \\
\mathrm{R}_{\mathrm{a}}(\mu \mathrm{m})\end{array}$ \\
\hline $\begin{array}{l}\text { AR } \\
\text { substrates }\end{array}$ & 78 & 95 & 32 & 63 & 145.7 & 0.9 \\
\hline $\begin{array}{l}\text { PDMS } \\
\text { coatings }\end{array}$ & 109 & 118 & 95 & 23 & 3.1 & 0.12 \\
\hline $\begin{array}{l}\text { Nano- } \\
\text { SiO } / \text { PDMS } \\
\text { coatings }\end{array}$ & 152 & 142 & 141 & 1 & 42 & 1.9 \\
\hline
\end{tabular}

\section{$217 \quad 3.2$ In-situ water condensation}

218 The sample substrates and coatings were exposed to high humidity levels (90-100\%

$219 \mathrm{RH}$ ) and temperatures were dropped to $1 \sim 4{ }^{\circ} \mathrm{C}$ range to carry out in situ water 220 condensation in a low vacuum chamber. The top and side views of micro-level water 221 condensation formed on AR-Al samples during in situ water condensation are shown 222 in Figure $3 a$ and $3 b$. It was observed that water condensed on AR substrates 223 randomly. The condensed water droplets were uniformly distributed but the droplet 224 size varied throughout the observed surface. 
225 The pattern of in-situ water condensation on superhydrophobic Nano-SiO $/$ PDMS 226 coatings was interesting and the surface morphology is shown in figure $4 \mathrm{a}$. It is clear 227 in Figure $4 \mathrm{~b}$ and $4 \mathrm{c}$ that micro-condensation on superhydrophobic surfaces is 228 imminent under high humidity conditions and three points could be drawn based on
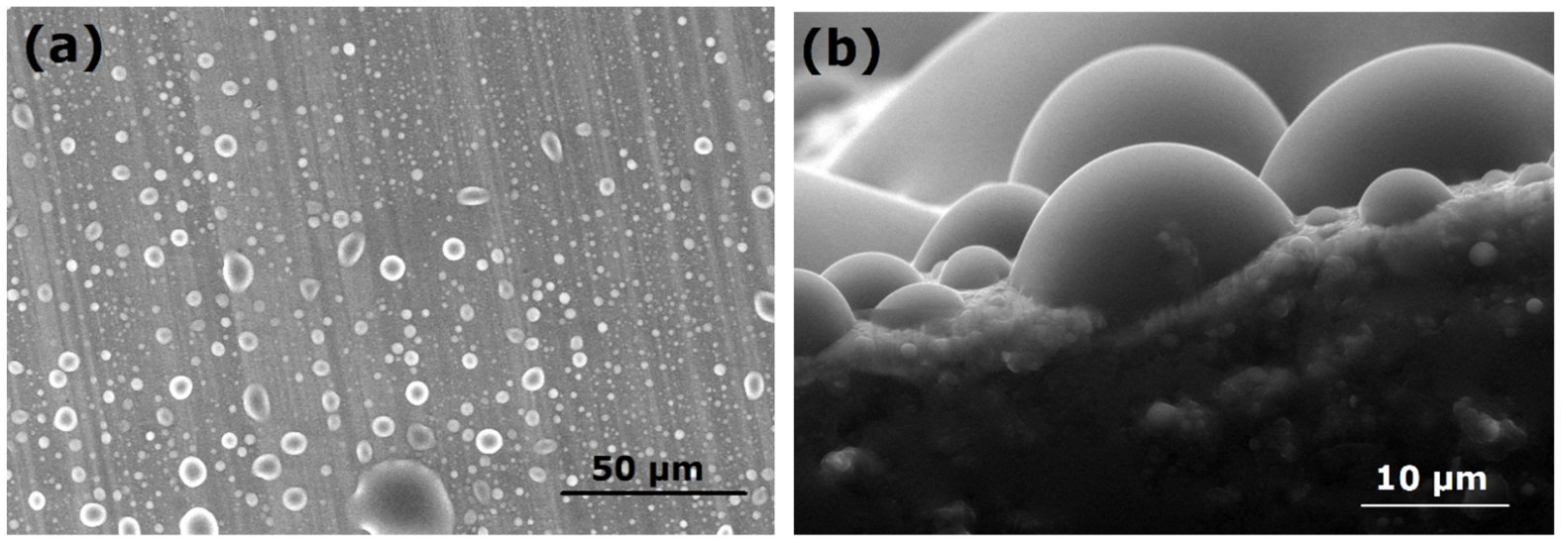

Figure 3: In-situ water condensation on AR substrates at $3{ }^{\circ} \mathrm{C}$ and $97 \%$ humidity

(a) Top view and (b) side view

229 the analysis. Firstly, the $\mathrm{Nano}-\mathrm{SiO}_{2} / \mathrm{PDMS}$ coatings had a rougher surface consisting 230 of the void valley along the surface and the in-situ water condensation results confirmed that the water condensation commenced along the rough asperities of the coatings. Thus, it can be assumed that the surface can only be entirely wetted when

233 the condensed droplets form a uniform water layer on the rough surfaces (Buoyancy), 234 i.e. wetting entire void valley and peaks. Ice grown from these condensed droplets will 

be interlocked in the rough asperities.
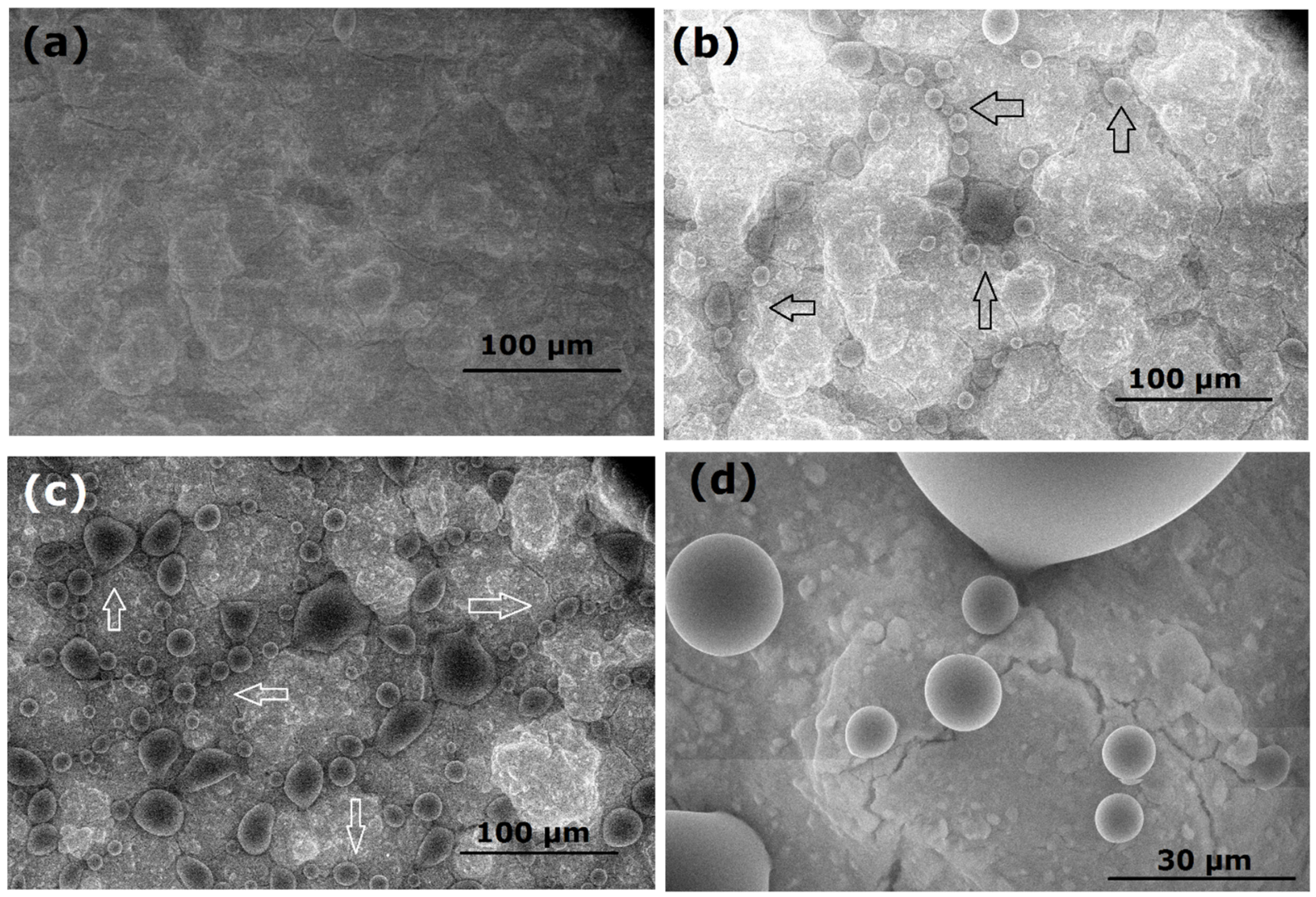

Figure 4: In-situ condensation on PDMS-Nano $\mathrm{SiO}_{2}$ coatings at $4{ }^{\circ} \mathrm{C}$ and $98 \%$ humidity (a) at start and after (b) 10 seconds, (c) 20 seconds (side position) and (d) magnified image at $100 \%$ humidity conditions

237 Secondly, the incorporation of hydrophobic silicon oxide nanoparticles induced the 238 formation of cracks over the surface and the cracks are prominent over the entire surface morphology. Examination of in-situ water condensation on these surfaces reveals that initiation of micro condensation of water started in the cracks as indicated

241 (arrows) in Figure 4a and 4b. It can be assumed that cracks act as nucleation seeds 242 for micro-condensation of water [18] and the micro-condensation compromise/nullify 243 the superhydrophobic ability of the material after formation of a thin layer of ice $[6,47]$. 244 Thirdly, the Nano-SiO 2 /PDMS coating surface demonstrated superhydrophobic 
performance at the micro level as shown in Figure $4 d$ and relatively large suspended water droplets in a much more spherical shape in comparison to AR substrates as shown in Figure 3a. The superhydrophobic ability of a material was also validated under high humidity conditions and in low vacuum (pressure) conditions at the microscale.

In-situ water condensation was also attempted on pristine hydrophobic PDMS coatings but no condensation was formed on these coatings under $100 \%$ humidity conditions. It is imperative to mention that the top view was adopted to validate in-situ water condensation and a thin layer of water might have formed on PDMS coatings which were not measured or observed due to equipment limitations and/or the transparent nature of the polymer coating.

\subsection{In-situ Icing tests}

257 Further to the water condensation tests, the temperature was dropped to $-5{ }^{\circ} \mathrm{C}$, to allow condensed water on the surface to be frozen for 30-60 minutes. To validate the anchoring of ice over surfaces, frozen ice was forced to melt/retract by increasing the temperature and ice retracting patterns were recorded.

Ice blocks formed on AR substrate are shown in Figure 5a. It is evident that ice was formed indiscriminately. Figure $5 b$ shows the grown ice over superhydrophobic coatings based on Nano- $\mathrm{SiO}_{2}$ /PDMS mixture and ice growth was much more consistent as compared with that on AR substrates. Layer by layer formation of ice could be attributed to the high humidity conditions. Layers of water condensation were frozen as they condense on the ice and coating/substrate surface. 
267 Preliminary results on the anchoring of ice over rough asperities surface are shown in

268 Figure 6. The ice formation over the superhydrophobic surface is still observed in

Figure $6 \mathrm{a}$, although the surface exhibited superhydrophobic behaviour at micro scale.

270 During the retracting process, which is intrinsically a shearing process [50, 51], some

271 ice stuck or anchored in the rough asperities at the highlighted area of the coating surface as shown in Figure $6 c-d$ and this is physically the first direct visual representation of the ice anchoring process. Through the process, the entire grown ice was retracted but the anchoring of ice over the surface was rigid and stubborn as
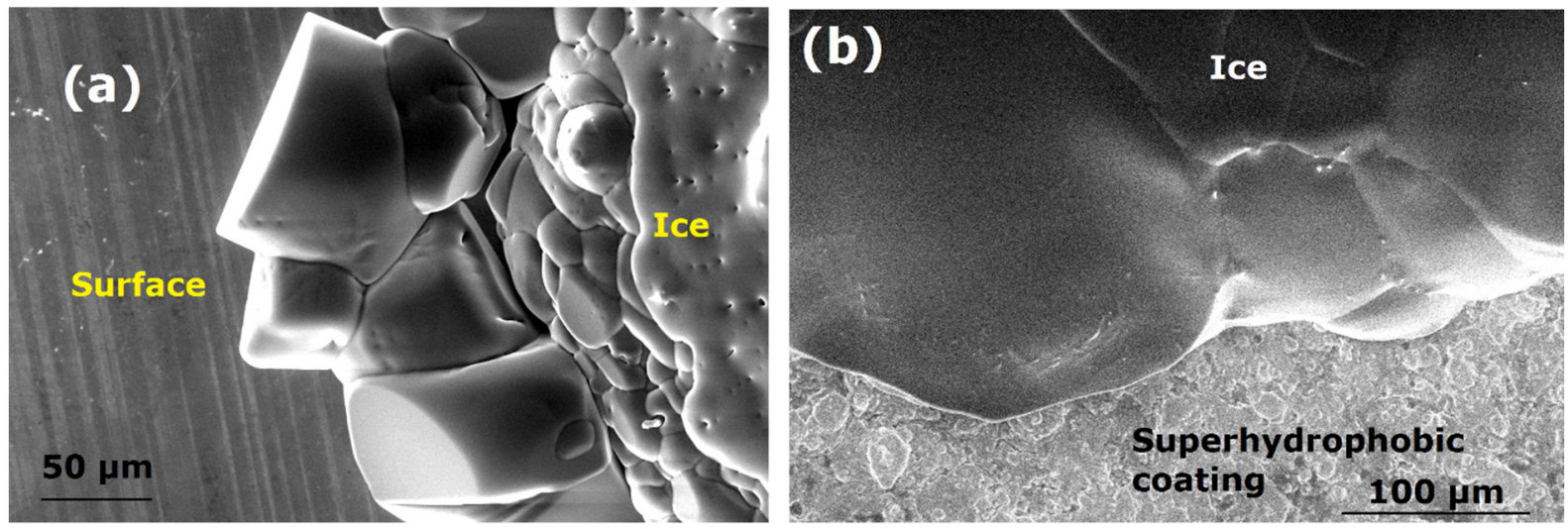

Figure 5: In-situ icing formation at $86 \%$ humidity and $-5^{\circ} \mathrm{C}$ on (a) AR substrates and (b) superhydrophobic coating

275 shown in Figure 6e-f.

276 From the observed results, it indicates that the ice adhesion strength on these rough surfaces will be significantly higher as compared to the surfaces with low surface roughness and it may damage the material and/or alter the surface morphology if this bulk ice is removed by means of shear force. This could be the main reason that superhydrophobic surface loses superhydrophobicity/icephobicity as either the water condensates in the void valleys under high humidity conditions and forms thin layer of ice $[6,47]$ or the shearing of this anchored ice distorts the rougher asperities on the surface and the superhydrophobicity could be mitigated as it is reliant on rough 

surface is nullified which has a domino effect on superhydrophobicity induced
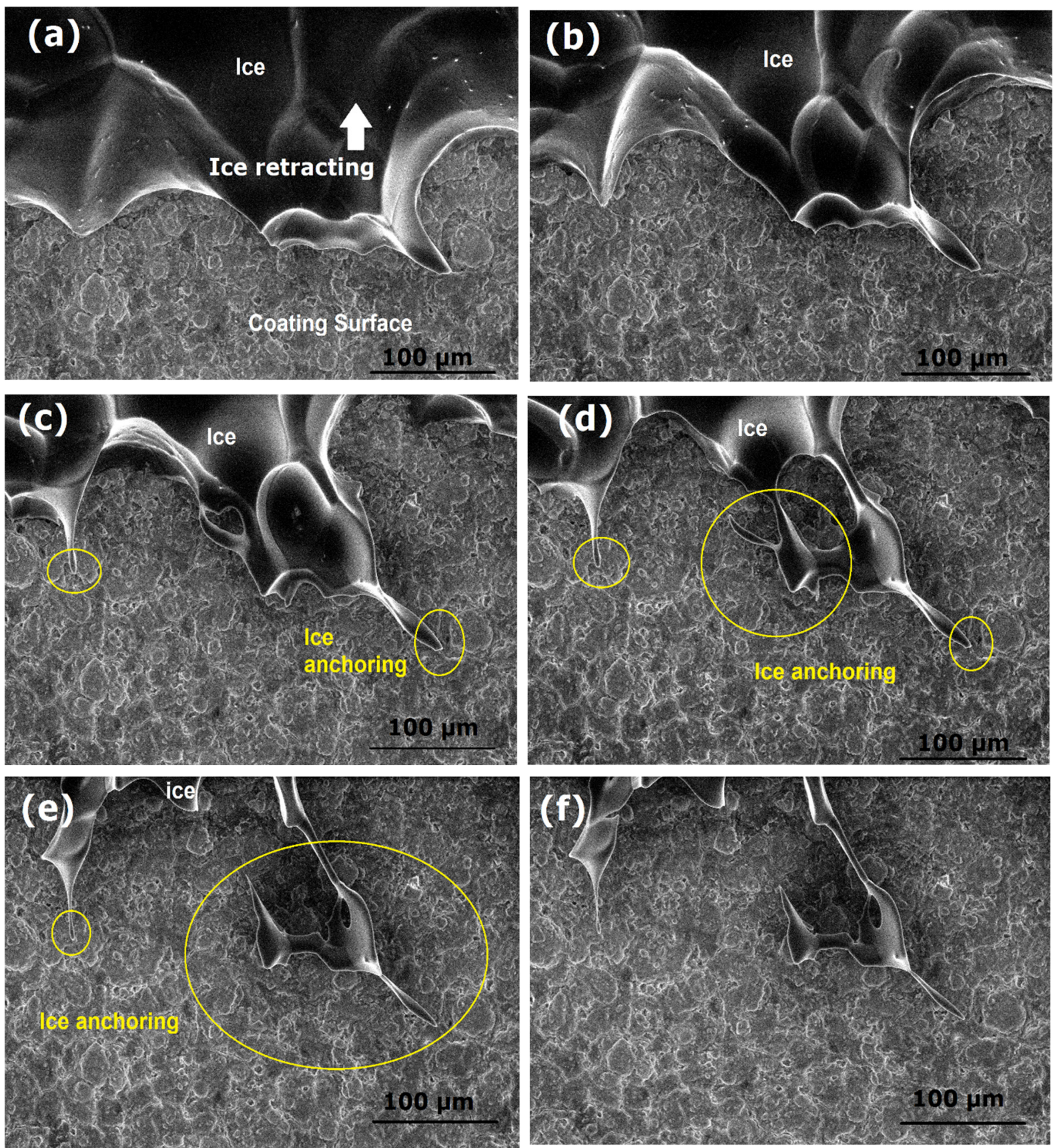

Figure 6: Ice anchoring mechanism on superhydrophobic surface after (a) 3, (b) 6, (c) 9, (d) 12, (e) 15 and (f) 18 seconds. icephobic performance.

287 The in-situ icing observations were further extended on as-received hydrophilic aluminum substrates as shown in Figure 7. Overview of the images indicates that the 
289 ice formed on the superhydrophobic surface was visually more solidified as a comparison to AR substrates. The ice retracting process (gradually increased in temperatures up to $-1^{\circ} \mathrm{C}$ and reduced humidity to around $80 \% \mathrm{RH}$ ) is shown in Figure $7 \mathrm{a}$ and $7 \mathrm{~b}$, the formed ice started to break apart in smaller ice segments. Further analysis reveals that the ice anchoring on AR substrates was much more widespread as compared to superhydrophobic surfaces. The intensity of ice anchoring on AR
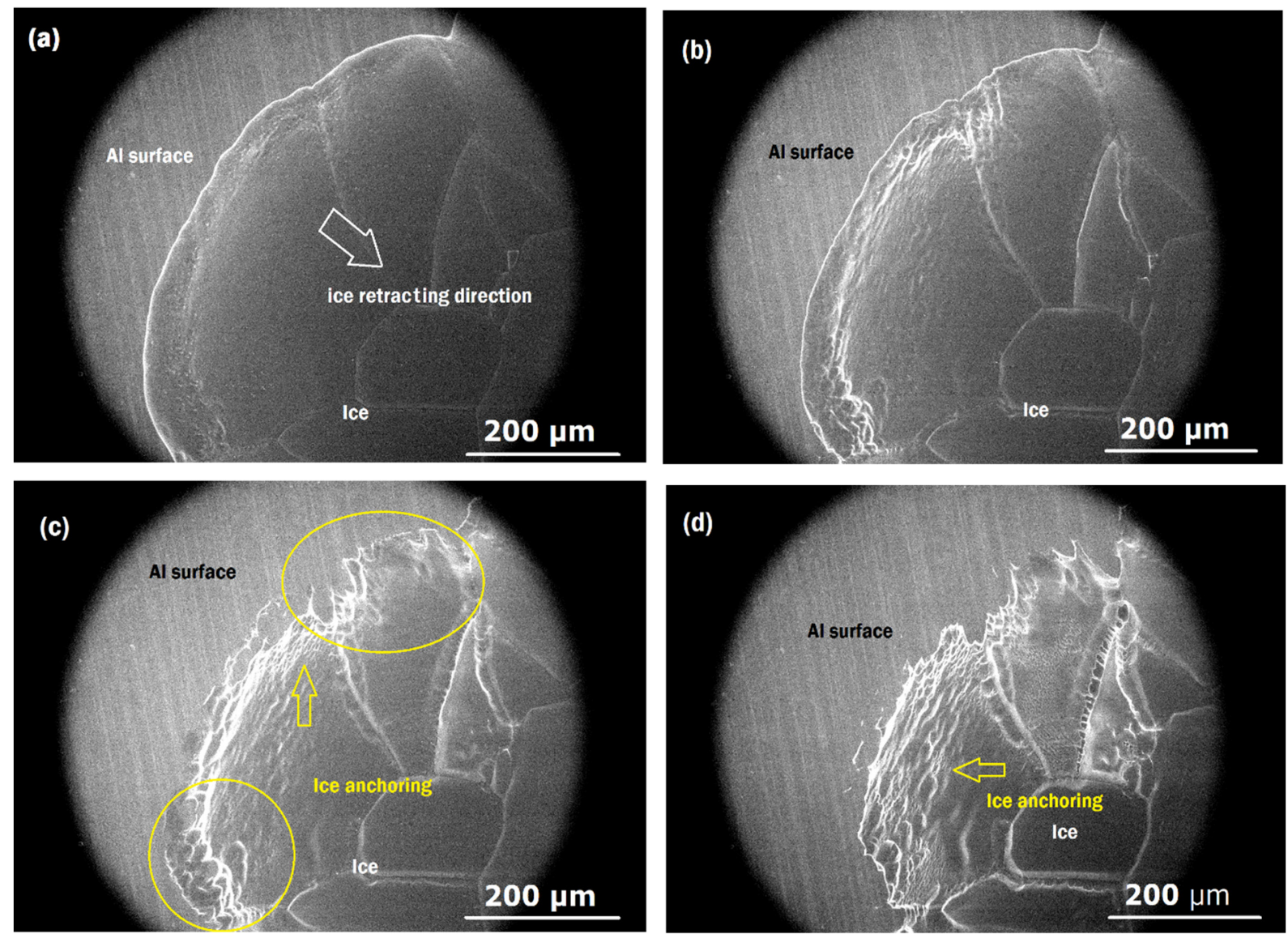

Figure 7: Ice anchoring mechanism on AR hydrophilic surfaces after (a) 3, (b) 6,

(c) 9 , and (d) 12 seconds.

samples was abundant as shown in Figure $7 c$ and $7 d$.

296 The evidence elaborated in this study is the first of direct validation of ice anchoring over retracting ice on different wettability surfaces. Many static icing studies in rough asperities were reported in the literature $[33,53,54]$, and a few dynamic icing studies were documented $[21,55]$ but no ice retracting study has been conducted at micro- 
level scale. Many researchers had argued and attempted to validate the ice anchoring over rough surfaces [56-58]. In-situ icing observation was also attempted on PDMS coatings where no water condensations were observed, thus no ice can be formed subsequently as the ice was formed from the condensed water on the surface. Preliminary results of ice anchoring were in good agreement with measured ice adhesion strength and indicated a strong linkage between centrifugal shearing of ice and anchoring mechanism on the surface rough voids. AR substrates showed enhanced ice anchoring and ice adhesion strength was much higher than superhydrophobic surfaces as listed in Table 1.

\subsection{Roughness dependence on ice adhesion strength}

It is clear from initial results that rough surface asperities provide anchoring points for the ice over the surface. To validate the ice anchoring mechanism and justify the effects on ice adhesion strength, the AR Al samples were treated by (1) grinding and polishing to smoothen ( $\left.R_{a} 0.05 \mu \mathrm{m}\right)$ and (2) sandblasting to roughen $\left(\sim R_{a} 1.2 \mu \mathrm{m}\right)$. Microstructural morphology of smoothened and roughened Al samples is shown in Figure $8 \mathrm{a}$ and $8 \mathrm{~b}$ respectively. The roughened substrates $\left(\sim \mathrm{R}_{a} 1.2 \mu \mathrm{m}\right)$ had disorder surface features as compare to the smoothened samples as shown in Figure $8 \mathrm{c}$ and $8 d$.

The hydrophobicity and icephobicity values of these substrates are listed in Table 2. The smoothened AR Al samples significantly reduced the ice adhesion strength on aluminum substrates. It is understood that rough asperities play a deciding role and ice anchoring over rougher surfaces is an influencing factor in icephobic studies. Interestingly, the $\mathrm{CAH}$ of the as-received and the smoothened aluminum substrates were similar but the ice adhesion strength varied by a factor of 11 . The ice over 
roughened substrates did not detach at the maximum rotation speed of centrifuge equipment, i.e. $4500 \mathrm{rpm}$ and the extrapolated results suggest that the formed ice had an adhesion strength of above $170 \mathrm{KPa}$. The results are in good agreement with Zaid et al studies [38] that different ice adhesion strength can be achieved with similar CAHs. It is further assumed that wettability of substrates does not play a prominent role in icephobicity studies and the substrates did not show any relevance in terms of water contact angles, either static or dynamic water contact angles. Thus, it can be
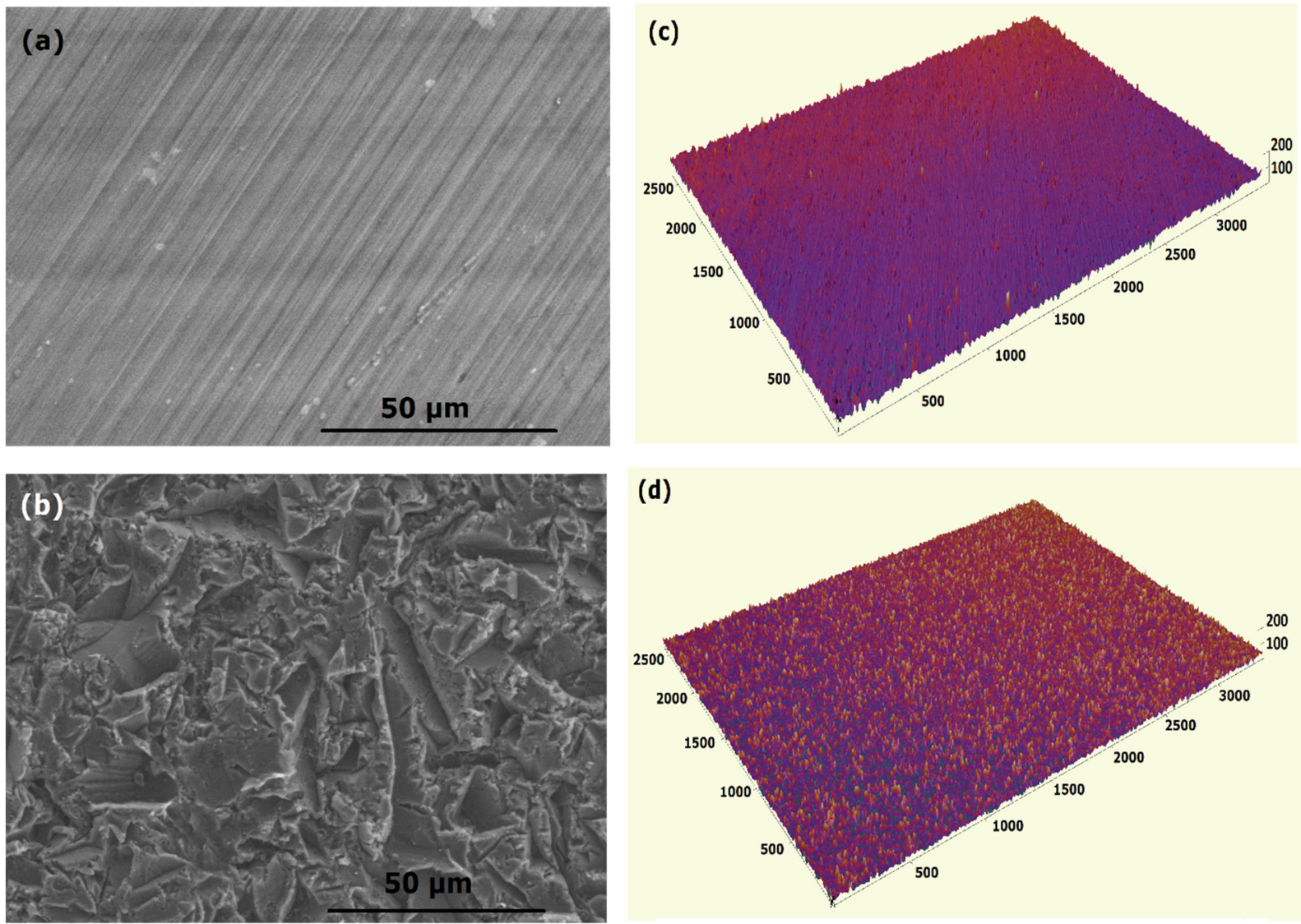

Figure 8: Microstructural images and 3D surface profile of (a) (c) smoothened and (b) (d) roughened aluminium substrates concluded that hydrophobicity is not entirely connected to icephobicity. 
Table 2: Wettability and icephobicity results of aluminum substrates.

\begin{tabular}{|l|l|l|l|l|l|l|}
\hline $\begin{array}{l}\text { Substrate } \\
\text { types }\end{array}$ & $\begin{array}{l}\text { Static } \\
\text { WCAs }\left(^{\circ}\right)\end{array}$ & $\begin{array}{l}\text { Advancing } \\
\text { WCAs }\left(^{\circ}\right)\end{array}$ & $\begin{array}{l}\text { Receding } \\
\text { WCAs }\left(^{\circ}\right)\end{array}$ & $\begin{array}{l}\text { CAH } \\
\left(^{\circ}\right)\end{array}$ & $\begin{array}{l}\text { Ice adhesion } \\
\text { strength }(\mathrm{KPa})\end{array}$ & $\begin{array}{l}\text { Roughness } \\
(\mu \mathrm{m})\end{array}$ \\
\hline As received & 78 & 95 & 32 & 63 & 145.7 & 0.9 \\
\hline Roughen & 54 & 56 & 14 & 42 & $>170^{*}$ & 1.2 \\
\hline Smoothen & 74 & 83 & 18 & 65 & 15.7 & 0.12 \\
\hline
\end{tabular}

337 *Extrapolation was based on the centrifugal force generated at the maximum speed of the centrifugal equipment while the detachment of ice did not occur.

\section{Conclusions}

340 The effect of rough asperities ice anchoring was long speculated in icephobicity studies but no direct validation was reported. Ice anchoring mechanism on surface voids was confirmed in the present work via in-situ icing observations and surface roughness directly contributed to ice anchoring. The superhydrophobic surface can only provide feasible ice protection before the formation of a thin layer of ice via microcondensation because the surface voids that induce superhydrophobicity also provides possible anchoring points for the ice. In either case, the superhydrophobicity induced icephobic performance of the coating surface is nullified. To validate this hypothesis, five different types of surface/coatings were investigated via in-situ water condensation and icing observations and the assumption was quantified using ice adhesion strength and evaluated based on surface rough asperities. coatings revealed that water condensed on the surface indiscriminately, however

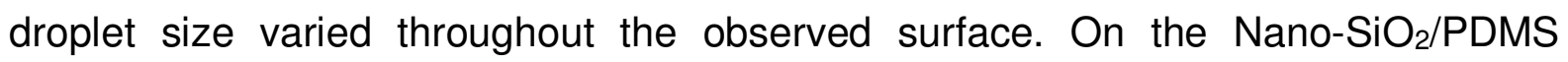


coatings, the water formed along rough asperities and surface cracks of the coatings which imparted ice anchoring and acted as seeds for heterogeneous ice nucleation. Ice grown from these condense droplets would require extra shearing force to remove (higher ice adhesion strength) as it would be interlocked (anchored) in rough asperities.

Strong visual evidence of the ice anchoring mechanism over surfaces has been obtained from the in-situ icing observation. The intensity of ice anchoring was dependent on surface asperities and the investigation revealed that the ice anchoring on AR-Al substrates ( $\left.R_{a} 0.9 \mu \mathrm{m}\right)$ was much more widespread as compared to Nano$\mathrm{SiO}_{2} /$ PDMS superhydrophobic coatings $\left(\sim \mathrm{R}_{\mathrm{a}} 1.9 \mu \mathrm{m}\right)$. To further validate the ice anchoring mechanism on different topographical surfaces, the AR-Al surface was smoothened $\left(\sim R_{a} 0.05 \mu \mathrm{m}\right)$ and roughened $\left(\sim R_{a} 1.2 \mu \mathrm{m}\right)$ using polishing and sandblasting, respectively. The $\mathrm{CAH}$ of the as-received and the smoothened aluminum substrates were similar but the ice adhesion strength varies by a factor of

11. The ice on the roughened substrates did not detach at the maximum rotation speed of centrifuge equipment (i.e. $4500 \mathrm{rpm}$ ) and the extrapolated results suggested that the ice adhesion strength was higher than $170 \mathrm{KPa}$. Interestingly, the surface

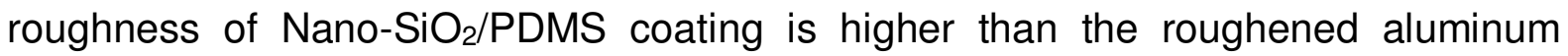
surface, however, the ice adhesion strength of polymer nanocomposite coating was lower. This signifies the combined effect of interfacial cavitation and superhydrophobicity induced icephobic performance. Overall results confirm that icephobicity is not entirely connected to hydrophobicity and ice anchoring occurs more widely on the rougher surface which significantly affects the ice adhesion strength. 


\section{Acknowledgment}

379

380

381

382

383

384

385

386

387

388

389

390

391

392

393

394

395

396

397

398

399

400

401

This work was supported by the studentship from Faculty of Engineering, University of Nottingham and has partially received funding from the CleanSky 2 Joint Undertaking under the European Union's Horizon 2020 research and innovation programme under grant agreement No CS2-AIR-GAM-2014-2015-O1. Cf. Art.29.4 of [A2]. The authors also thank Dr. Barbara Turnbull for helping with ice adhesion strength test and acknowledge the use of facilities at Nanoscale and Microscale Research Centre of University of Nottingham supported by Engineering and Physical Sciences Research Council [grant number EP/L022494/1].

\section{Declaration of interest statement}

Declarations of interest: none.

\section{References}

[1] S. Jung, M. Dorrestijn, D. Raps, A. Das, C.M. Megaridis, D. Poulikakos, Are superhydrophobic surfaces best for icephobicity?, Langmuir, 27 (2011) 3059-3066.

[2] Y. Wang, J. Xue, Q. Wang, Q. Chen, J. Ding, Verification of icephobic/anti-icing properties of a superhydrophobic surface, ACS applied materials \& interfaces, 5 (2013) 3370-3381.

[3] R. Jafari, R. Menini, M. Farzaneh, Superhydrophobic and icephobic surfaces prepared by RF-sputtered polytetrafluoroethylene coatings, Applied Surface Science, 257 (2010) 1540-1543.

[4] M. Nosonovsky, V. Hejazi, Why superhydrophobic surfaces are not always icephobic, ACS nano, 6 (2012) 8488-8491.

[5] G. Fang, A. Amirfazli, Understanding the anti-icing behavior of superhydrophobic surfaces, Surface Innovations, 2 (2014) 94-102. 
402

403

404

405

406

407

408

409

410

411

412

413

414

415

416

417

418

419

420

421

422

423

424

425

[6] A.J. Meuler, G.H. McKinley, R.E. Cohen, Exploiting topographical texture to impart icephobicity, ACS nano, 4 (2010) 7048-7052.

[7] K.K. Varanasi, M. Hsu, N. Bhate, W. Yang, T. Deng, Spatial control in the heterogeneous nucleation of water, Applied Physics Letters, 95 (2009) 094101.

[8] A.J. Meuler, J.D. Smith, K.K. Varanasi, J.M. Mabry, G.H. McKinley, R.E. Cohen, Relationships between water wettability and ice adhesion, ACS applied materials \& interfaces, 2 (2010) 3100-3110.

[9] V. Hejazi, K. Sobolev, M. Nosonovsky, From superhydrophobicity to icephobicity: forces and interaction analysis, Scientific reports, 3 (2013) 2194.

[10] A. Tuteja, W. Choi, M. Ma, J.M. Mabry, S.A. Mazzella, G.C. Rutledge, G.H. McKinley, R.E. Cohen, Designing superoleophobic surfaces, Science, 318 (2007) 1618-1622.

[11] A. Lafuma, D. Quéré, Superhydrophobic states, Nature materials, 2 (2003) 457.

[12] A. Cassie, S. Baxter, Wettability of porous surfaces, Transactions of the Faraday society, 40 (1944) 546-551.

[13] S. Herminghaus, Roughness-induced non-wetting, EPL (Europhysics Letters), 52 (2000) 165.

[14] D. Öner, T.J. McCarthy, Ultrahydrophobic surfaces. Effects of topography length scales on wettability, Langmuir, 16 (2000) 7777-7782.

[15] Z. Yoshimitsu, A. Nakajima, T. Watanabe, K. Hashimoto, Effects of surface structure on the hydrophobicity and sliding behavior of water droplets, Langmuir, 18 (2002) 5818-5822.

[16] S. Shibuichi, T. Onda, N. Satoh, K. Tsujii, Super water-repellent surfaces resulting from fractal structure, The Journal of Physical Chemistry, 100 (1996) 19512-19517. 
[17] T. Onda, S. Shibuichi, N. Satoh, K. Tsujii, Super-water-repellent fractal surfaces, 427 Langmuir, 12 (1996) 2125-2127.

[18] P. Kim, T.-S. Wong, J. Alvarenga, M.J. Kreder, W.E. Adorno-Martinez, J. Aizenberg, Liquid-infused nanostructured surfaces with extreme anti-ice and anti-frost performance, ACS nano, 6 (2012) 6569-6577.

[19] M. Zou, S. Beckford, R. Wei, C. Ellis, G. Hatton, M. Miller, Effects of surface roughness and energy on ice adhesion strength, Applied Surface Science, 257 (2011) 3786-3792.

[20] P. Eberle, M.K. Tiwari, T. Maitra, D. Poulikakos, Rational nanostructuring of surfaces for extraordinary icephobicity, Nanoscale, 6 (2014) 4874-4881.

[21] L. Mishchenko, B. Hatton, V. Bahadur, J.A. Taylor, T. Krupenkin, J. Aizenberg, Design of ice-free nanostructured surfaces based on repulsion of impacting water droplets, ACS nano, 4 (2010) 7699-7707.

[22] J. Liu, Z.A. Janjua, M. Roe, F. Xu, B. Turnbull, K.-S. Choi, X. Hou, Superhydrophobic/icephobic coatings based on silica nanoparticles modified by selfassembled monolayers, Nanomaterials, 6 (2016) 232.

[23] M. Hancer, H. Arkaz, A facile fabrication of superhydrophobic nanocomposite coating with contact angles approaching the theoretical limit, Applied Surface Science, 354 (2015) 342-346.

[24] L. Cao, A.K. Jones, V.K. Sikka, J. Wu, D. Gao, Anti-icing superhydrophobic coatings, Langmuir, 25 (2009) 12444-12448. [25] K.K. Varanasi, T. Deng, J.D. Smith, M. Hsu, N. Bhate, Frost formation and ice adhesion on superhydrophobic surfaces, Applied Physics Letters, 97 (2010) 234102. 
[26] K.A. Wier, T.J. McCarthy, Condensation on ultrahydrophobic surfaces and its

450

451

452

453

454

455

456

457

458

459

460

461

462

463

464

465

466

467

468

469

470

471

472

473

effect on droplet mobility: ultrahydrophobic surfaces are not always water repellant, Langmuir, 22 (2006) 2433-2436.

[27] K.R. Murphy, W.T. McClintic, K.C. Lester, C.P. Collier, J.B. Boreyko, Dynamic defrosting on scalable superhydrophobic surfaces, ACS applied materials \& interfaces, 9 (2017) 24308-24317.

[28] P. Tourkine, M. Le Merrer, D. Quéré, Delayed freezing on water repellent materials, Langmuir, 25 (2009) 7214-7216.

[29] A. Kirillova, L. Ionov, I.V. Roisman, A. Synytska, Hybrid Hairy Janus Particles for Anti-Icing and De-Icing Surfaces: Synergism of Properties and Effects, Chem. Mater, 28 (2016) 6995-7005.

[30] S. Ozbay, C. Yuceel, H.Y. Erbil, Improved icephobic properties on surfaces with a hydrophilic lubricating liquid, ACS applied materials \& interfaces, 7 (2015) 2206722077.

[31] J. Zhou, S. Xu, S. Huang, X. Meng, J. Sheng, H. Zhang, J. Li, Y. Sun, E. Boateng, Tensile properties and microstructures of a 2024-T351 aluminum alloy subjected to cryogenic treatment, Metals, 6 (2016) 279.

[32] Z. Wang, A.A. Volinsky, N.D. Gallant, Crosslinking effect on polydimethylsiloxane elastic modulus measured by custom-built compression instrument, Journal of Applied Polymer Science, 131 (2014).

[33] K. Li, S. Xu, W. Shi, M. He, H. Li, S. Li, X. Zhou, J. Wang, Y. Song, Investigating the effects of solid surfaces on ice nucleation, Langmuir, 28 (2012) 10749-10754.

[34] K. Li, S. Xu, J. Chen, Q. Zhang, Y. Zhang, D. Cui, X. Zhou, J. Wang, Y. Song, Viscosity of interfacial water regulates ice nucleation, Applied Physics Letters, 104 (2014) 101605. 
[35] S. Ozbay, H.Y. Erbil, Ice accretion by spraying supercooled droplets is not dependent on wettability and surface free energy of substrates, Colloids and Surfaces

A: Physicochemical and Engineering Aspects, 504 (2016) 210-218.

477 [36] J. Liu, G. Zong, L. He, Y. Zhang, C. Liu, L. Wang, Effects of fumed and mesoporous silica nanoparticles on the properties of sylgard 184 polydimethylsiloxane, Micromachines, 6 (2015) 855-864.

[37] R.M. Fillion, A. Riahi, A. Edrisy, Design factors for reducing ice adhesion, Journal of Adhesion Science and Technology, (2017) 1-14.

[38] Z.A. Janjua, B. Turnbull, K.-L. Choy, C. Pandis, J. Liu, X. Hou, K.-S. Choi,

Performance and durability tests of smart icephobic coatings to reduce ice adhesion, Applied Surface Science, 407 (2017) 555-564.

[39] S. Kulinich, M. Farzaneh, How wetting hysteresis influences ice adhesion strength on superhydrophobic surfaces, Langmuir, 25 (2009) 8854-8856.

[40] S. Kulinich, M. Farzaneh, Ice adhesion on super-hydrophobic surfaces, Applied

Surface Science, 255 (2009) 8153-8157.

[41] S. Kulinich, M. Farzaneh, On ice-releasing properties of rough hydrophobic coatings, Cold Regions Science and Technology, 65 (2011) 60-64.

[42] H. Zhu, Z. Guo, W. Liu, Adhesion behaviors on superhydrophobic surfaces,

Chemical Communications, 50 (2014) 3900-3913.

[43] J. Seyfi, S.H. Jafari, H.A. Khonakdar, G.M.M. Sadeghi, G. Zohuri, I. Hejazi, F.

Simon, Fabrication of robust and thermally stable superhydrophobic nanocomposite coatings based on thermoplastic polyurethane and silica nanoparticles, Applied Surface Science, 347 (2015) 224-230. graphene films, Langmuir, 25 (2009) 11078-11081. 
[45] D. Janssen, R. De Palma, S. Verlaak, P. Heremans, W. Dehaen, Static solvent contact angle measurements, surface free energy and wettability determination of various self-assembled monolayers on silicon dioxide, Thin Solid Films, 515 (2006) 1433-1438.

[46] D.K. Owens, R. Wendt, Estimation of the surface free energy of polymers, Journal of applied polymer science, 13 (1969) 1741-1747.

[47] J. Chen, Z. Luo, Q. Fan, J. Lv, J. Wang, Anti-Ice Coating Inspired by Ice Skating, Small, 10 (2014) 4693-4699.

[48] C. Stamatopoulos, J. Hemrle, D. Wang, D. Poulikakos, Exceptional anti-icing performance of self-impregnating slippery surfaces, ACS applied materials \& interfaces, 9 (2017) 10233-10242.

[49] S. Bengaluru, K. Kripa, Mechanism of Frost Formation on Lubricant-Impregnated Surfaces, Langmuir, (2013). [50] L. Gao, T.J. McCarthy, Wetting 101º, Langmuir, 25 (2009) 14105-14115.

[51] B. Liu, K. Zhang, C. Tao, Y. Zhao, X. Li, K. Zhu, X. Yuan, Strategies for anti-icing: low surface energy or liquid-infused?, RSC Advances, 6 (2016) 70251-70260. [52] P. Hao, C. Lv, X. Zhang, Freezing of sessile water droplets on surfaces with various roughness and wettability, Applied physics letters, 104 (2014) 161609. [53] Y. He, C. Jiang, X. Cao, J. Chen, W. Tian, W. Yuan, Reducing ice adhesion by 518 hierarchical micro-nano-pillars, Applied Surface Science, 305 (2014) 589-595. [54] S. Bengaluru Subramanyam, V. Kondrashov, J.r. Rühe, K.K. Varanasi, Low ice adhesion on nano-textured superhydrophobic surfaces under supersaturated conditions, ACS applied materials \& interfaces, 8 (2016) 12583-12587. 
[55] Z. Jin, D. Sui, Z. Yang, The impact, freezing, and melting processes of a water

523 droplet on an inclined cold surface, International journal of heat and mass transfer, 90

524 (2015) 439-453.

525 [56] J.M. Campbell, F.C. Meldrum, H.K. Christenson, Is ice nucleation from 526 supercooled water insensitive to surface roughness?, The Journal of Physical 527 Chemistry C, 119 (2015) 1164-1169.

528 [57] G. Momen, R. Jafari, M. Farzaneh, Ice repellency behaviour of superhydrophobic 529 surfaces: effects of atmospheric icing conditions and surface roughness, Applied 530 Surface Science, 349 (2015) 211-218.

531 [58] Q.T. Fu, E.J. Liu, P. Wilson, Z. Chen, Ice nucleation behaviour on sol-gel coatings 532 with different surface energy and roughness, Physical Chemistry Chemical Physics, $53317(2015)$ 21492-21500. 